\title{
Análise Da Taxa De Infecção Hospitalar Em Um Hospital Universitário Do Rio Grande Do Sul
}

\author{
Fernandes, V.F.; Valente, A.P.; Butzke, B.; Jimenez, L.F.; Cittolin-Santos, G.F.; \\ Kipper, C.E.; Kluck, M.M.;
}

Apresentador: Vinícius Fornari Fernandes

\section{Resumo}

Introdução: A taxa de infecção hospitalar é um dos fatores prioritários para promover a segurança do paciente e avaliação da qualidade assistencial. A análise de seus indicadores é necessária a fim de se buscar medidas de controle em tempo hábil a ser tomado tanto pelas instituições, quanto pelos órgãos orientadores. Este trabalho objetiva analisar os dados referentes à taxa de infecção hospitalar no Hospital de Clínicas de Porto Alegre (HCPA) no período de 2001 até 2013. Métodos: Foi realizada uma análise retrospectiva da taxa geral de infecção hospitalar no período de Janeiro de 2001 até Dezembro de 2013, a partir de dados epidemiológicos obtidos no sistema de informações gerenciais do HCPA, associado ao embasamento dos critérios nacionais de infecções hospitalares da ANVISA e dos critérios do Centers for Disease Control and Prevention (CDC). O cálculo da média e do desvio padrão dos dados colhidos e a análise de tendência de infecção hospitalar foram realizados no Software Excel 2013. Resultados: A taxa geral de infecção hospitalar média no período

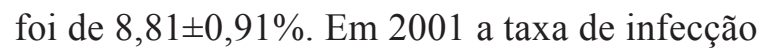
foi de 9,51\%, já em 2013 foi de 7,13\%, ou seja, houve redução relativa da taxa de infecção hospitalar no período de $25 \%$. Além disso, é possível observar tendência à diminuição na taxa de infecção hospitalar ao longo do tempo $(\mathrm{y}=-0.202 \mathrm{x}+10.271$ $\left.\mathrm{R}^{2}=0.7566\right)$. Conclusão: A análise das taxas de infecções hospitalares é fundamental para avaliar o risco dos serviços de saúde e dessa maneira prevenir e intervir sobre eventos potencialmente infecciosos e sobre a segurança ao paciente. A introdução e difusão do uso álcool gel nas mãos, a criação de protocolos de prevenção de infecção baseados no perfil dos pacientes internados, o uso criterioso de antibióticos e o constante treinamento e envolvimento das equipes assistenciais são algumas das medidas efetivadas na última década no HCPA. A redução das taxas de infecção hospitalar evidencia a preocupação desta instituição com exercício das melhores práticas assistenciais, associado a maior adesão dos profissionais da área da saúde, a fim de otimizar a redução das taxas de infecção hospitalar.

\section{Referência:}

Fernandes, V.F.; Valente, A.P.; Butzke, B.; Jimenez, L.F.; Cittolin-Santos, G.F.; Kipper, C.E.; Kluck, M.M.;. Análise Da Taxa De Infecção Hospitalar Em Um Hospital Universitário Do Rio Grande Do Sul. In: Il Congresso Brasileiro de Medicina Hospitalar - II CBMH [= Blucher Medical Proceedings, vol.1, num.5] São Paulo: Editora Blucher, 2014. p.15

DOI 10.5151/medpro-II-cbmh-006 